\title{
The association of low complement with disease activity in systemic sclerosis: a prospective cohort study
} \author{
James Esposito ${ }^{1,2}$, Zoe Brown², Wendy Stevens ${ }^{2}$, Joanne Sahhar ${ }^{3,4}$, Candice Rabusa ${ }^{2}$, Jane Zochling ${ }^{5}$, Janet Roddy ${ }^{6}$,
} Jennifer Walker ${ }^{7}$, Susanna M. Proudman ${ }^{8,9}$ and Mandana Nikpour ${ }^{1,2^{*}}$

\begin{abstract}
Background: In some rheumatic diseases such as systemic lupus erythematosus (SLE), low serum complement ('hypocomplementaemia') is a feature of active disease. However, the role of hypocomplementaemia in systemic sclerosis (SSc) is unknown. We sought to determine the frequency, clinical associations and relationship to disease activity of hypocomplementaemia in SSC.

Methods: The study included 1140 patients fulfilling the 2013 American College of Rheumatology criteria for SSc. Demographic, serological and clinical data, obtained prospectively through annual review, were analysed using univariable methods. Linear and logistic regression, together with generalised estimating equations, were used to determine the independent correlates of hypocomplementaemia ever, and at each visit, respectively.

Results: At least one episode of hypocomplementaemia (low C3 and/or low C4) occurred in $24.1 \%$ of patients over 1893 visits; these patients were more likely to be seropositive for anti-ribonucleoprotein $(\mathrm{OR}=3.8, p=0.002)$, anti-Ro (OR $=2.2, p=0.002)$, anti-Smith $(\mathrm{OR}=6.3, p=0.035)$ and anti-phospholipid antibodies $(\mathrm{OR}=1.4, p=0.021)$ and were more likely to display features of overlap connective tissue disease, in particular polymyositis (OR $=16.0$, $p=0.012$ ). However, no association was found between hypocomplementaemia and either the European Scleroderma Study Group disease activity score or any of its component variables (including erythrocyte sedimentation rate) in univariate analysis. Among patients with SSc overlap disease features, those who were hypocomplementaemic were more likely to have digital ulcers $(\mathrm{OR}=1.6, p=0.034)$, tendon friction rubs ( $\mathrm{OR}=2.4, p=0.037)$, forced vital capacity $<80 \%$ predicted $(\mathrm{OR}=2.9, p=0.008)$ and lower body mass index $(\mathrm{BMI})(\mathrm{OR}$ for $\mathrm{BMI}=0.9, p<0.0005)$ at that visit, all of which are features associated with SSc disease activity and/or severity.
\end{abstract}

Conclusions: While hypocomplementaemia is not associated with disease activity in patients with non-overlap SSc, it is associated with some features of increased SSC disease activity in patients with overlap disease features.

Keywords: Systemic sclerosis, Complement, Disease activity

\section{Background}

Systemic sclerosis (SSc) or scleroderma is a systemic autoimmune disorder of unknown aetiology associated with substantial morbidity and mortality [1]. The hallmark pathological features of SSc are vasculopathy, inflammation and progressive perivascular and interstitial

\footnotetext{
* Correspondence: m.nikpour@unimelb.edu.au

'Department of Medicine, The University of Melbourne at St Vincent's

Hospital (Melbourne), 41 Victoria Parade, Fitzroy, VIC 3065, Australia

${ }^{2}$ Department of Rheumatology, St Vincent's Hospital (Melbourne), 41 Victoria

Parade, Fitzroy, VIC 3065, Australia

Full list of author information is available at the end of the article
}

fibrosis [2]. Prominent clinical manifestations of SSc include skin thickening, Raynaud's phenomenon, digital ulcers, gut involvement (gastro-oesophageal reflux disease, intestinal hypomotility and pseudoobstruction), pulmonary arterial hypertension (PAH), interstitial lung disease (ILD) and renal crisis [3]. An 'SSc overlap' syndrome is considered present when a patient with SSc also has clinical and/or serological evidence of one or more of systemic lupus erythematosus (SLE), polymyositis, Sjögren's syndrome or rheumatoid arthritis [2]. 
As in other systemic autoimmune diseases, disease activity leads to irreversible organ damage. Activity reflects features of the disease process that vary over time and are potentially reversible with intervention or spontaneously. Quantifying active disease would assist clinicians in assessment and management of disease activity, which might in turn prevent damage and improve outcomes [4]. Assessing disease activity is necessary for staging the disease and predicting prognosis. It is also useful for distinguishing between those patients requiring aggressive treatment and those for whom symptomatic treatment may be sufficient, as well as for monitoring response to treatment.

In certain rheumatic diseases characterised by distinct episodes of inflammation, such as SLE, validated measures already exist to assist clinicians in the assessment of disease activity [5]. There have been several endeavours to develop disease activity criteria for SSc. The European Scleroderma Study Group (EScSG) activity index was constructed on the basis of evaluation of features of a large multicentre cohort of patients with SSc, compared with a gold standard of disease activity assessment by three experts [6-8]. Univariate analysis of symptoms, signs and laboratory tests was performed to select single items that were significantly associated with the consensus disease activity score. The final index comprised ten components, including clinical features such as arthritis, modified Rodnan skin score (MRSS) and digital ulcers; patientreported changes in cardiopulmonary, skin and vascular symptoms; and erythrocyte sedimentation rate (ESR) $[6,7]$. Hypocomplementaemia was also included $[7,8]$, but it is not yet known whether low complement is truly a marker of disease activity in SSc.

Various candidate serological markers have been proposed to be related to disease activity in SSc, such as soluble interleukin-2 receptor (sIL-2R), type III procollagen aminopeptide (PIIINP) and von Willebrand factor propeptide [9-11]. However, in patients with SSc, changes in these markers have not been reflected in clinical responses to therapy. For example, chlorambucil administration was followed by normalization of sIL-2R levels but had no effect on disease activity [12]. Similarly, $\alpha$-interferon administration resulted in a decrease in PIIINP levels without any improvement in disease activity assessed using a validated skin score, grip strength, measurements of digital contractures, Ritchie index, assessment of muscle weakness, and tendon friction rubs [13].

Low serum complement is a candidate serological marker of disease activity in SSc. Aberrant activation of the complement system is implicated in the pathogenesis of a number of systemic autoimmune disorders [14]. For example, in SLE, immune complex formation triggers the complement cascade via the classical pathway, and the resulting low concentrations of complement components $\mathrm{C} 3$ and $\mathrm{C} 4$ are found in many patients with active and severe SLE $[15,16]$. It has been shown that the manifestations of SSc are due to derangements in both innate and adaptive immunity. The association of Tolllike receptor (TLR) signalling variations with SSc suggests that TLR pathways, and hence complement activation and consumption, may play a role in the pathogenesis of SSc [17]. However, unlike SLE, wherein low serum complement is a validated measure of disease activity, the relationship between hypocomplementaemia and disease activity in SSc is less clear.

The aim of this study was to evaluate the frequency of hypocomplementaemia in a cohort of patients with SSc and to evaluate which clinical and serological features are significantly associated with hypocomplementaemia. We hypothesised that hypocomplementaemia is associated with clinical features of disease activity such as arthritis, tendon friction rubs, low body mass index (BMI) (a marker of severe SSc gastrointestinal involvement) and low forced vital capacity (FVC) (a measure of severe ILD).

\section{Methods}

\section{Patients}

Patients were recruited from the Australian Scleroderma Cohort Study, a prospective multi-centre cohort study of risk factors for clinically important outcomes in SSc. All patients fulfilled the 2013 American College of Rheumatology classification criteria for SSc [18]. Patients were recruited from multiple Australian centres that specialize in the care of patients with SSc, which included St Vincent's Hospital and Monash Medical Centre, Victoria; Royal Prince Alfred and St George Hospitals, New South Wales; Sunshine Coast Rheumatology and Prince Charles Hospital, Queensland; Royal Adelaide Hospital, The Queen Elizabeth Hospital and Flinders Medical Centre, South Australia; Royal Perth Hospital, Western Australia; and Menzies Institute for Medical Research, Tasmania. The study was approved by the human research ethics committees of each of the participating centres.

Consent was obtained from all patients prior to the collection of demographic and disease-related data (including clinical and laboratory data) according to a standardized protocol at recruitment and at each subsequent annual review. Patients were included if they had at least one annual visit where complement levels had been recorded, along with demographic, clinical and laboratory data (see below).

\section{Measurement of complement levels}

C3 and C4 levels were measured prospectively at each annual visit in the laboratories of each of the recruiting centres using sera obtained at recruitment and at each 
subsequent review by performing nephelometry. Complement levels were defined as being either normal or low according to local laboratory standards. Patient visits were then recorded as normocomplementaemic if the patient had a normal $\mathrm{C} 3$ and $\mathrm{C} 4$ result or hypocomplementaemic if the patient had a low $\mathrm{C} 3$ and/or C4.

\section{Demographic, clinical and laboratory variables}

All data were collected prospectively.

\section{Data collected at recruitment}

Demographic data collected at recruitment included age at disease onset and age at recruitment. Disease onset was defined according to the age at which the first nonRaynaud's phenomenon manifestation of SSc occurred. Sex and race were also recorded; race was categorized as white, Asian, Aboriginal or Torres Strait Islander, or other. Disease duration was defined as the time elapsed from disease onset to recruitment, and length of follow-up was defined as the time elapsed from recruitment until the date at which the data were censored for analysis.

Disease-related data gathered included SSc subtype (diffuse or limited) defined according to the LeRoy criteria [19]. The autoantibody status of each patient was determined at recruitment using immunofluorescence for antinuclear antibodies and commercially available assays for antibodies to extractable nuclear antigens Scl-70, Jo-1, ribonucleoprotein (RNP), Ro, La, Smith (Sm), and polymyositis scleroderma (PM-Scl) (enzyme-linked immunosorbent assay [ELISA]; ORGENTEC Diagnostika, Mainz, Germany); antibodies to double-stranded DNA (anti-dsDNA) (Amerlex radioimmunoassay; Trinity Biotech, Bray, Ireland); antibodies to RNA polymerase III (QUANTA Lite RNA Pol III; Inova Diagnostics, San Diego, CA, USA; and MBL Anti-RNA Pol III; MBL International, Woburn, MA, USA); anti-neutrophil cytoplasmic antibodies (ANCA), including proteinase-3 or myeloperoxidase specificity (ELISA; ORGENTEC Diagnostika); rheumatoid factor and anti-phospholipid antibodies, including anti-cardiolipin antibodies (Vital Diagnostics, Bella Vista, Australia); and anti- $\beta_{2}$-glycoprotein antibodies (ELISA; ORGENTEC Diagnostika).

\section{Data collected at each annual visit}

Disease manifestations were recorded at each visit. Raynaud's phenomenon was defined on the basis of characteristic colour changes in the extremities. The presence of puffy digits (scleroderma), tendon friction rubs, synovitis and muscle atrophy was based on examination findings of the patient's rheumatologist. The MRSS [20] was also calculated and recorded at each visit.

Persistent sicca symptoms were defined as dry eyes or dry mouth at two or more annual visits. Gastrointestinal symptoms, including reflux requiring treatment with proton pump inhibitor, dysphagia, post-prandial bloating, vomiting, diarrhoea (more than three motions per day), constipation (fewer than one motion per day) or anal incontinence (faecal soiling) not due to other causes, were also recorded. Gastric antral vascular ectasia (GAVE) and oesophageal stricture were defined on the basis of endoscopy. Bowel dysmotility was defined on the basis of barium and nuclear medicine studies, antibiotic response and characteristic symptoms. Pseudo-obstruction was defined as the presence of clinical features suggestive of intestinal obstruction in the absence of an anatomical lesion.

Myocardial disease was defined on the basis of endomyocardial biopsy or as the presence of conduction defects, arrhythmias, and right ventricular or left ventricular dysfunction on echocardiography in the absence of other causes. Pericardial effusions were defined on the basis of an echocardiogram showing other than a small $(<1-\mathrm{cm}$ thickness), non-significant pericardial effusion.

PAH was defined on the basis of right heart catheterization as a mean pulmonary arterial pressure $\geq 25$ $\mathrm{mmHg}$ and a pulmonary arterial wedge pressure $\leq 15$ $\mathrm{mmHg}$. The 6-minute walk distance was also recorded each year as a measure of PAH severity.

ILD was defined on the basis of characteristic changes visualised by chest high-resolution computed tomography. Pulmonary function tests were performed at each annual visit, and forced vital capacity (FVC) and diffusing capacity of the lung for carbon monoxide (DLCO) were recorded as percent predicted values, with FVC used as a measure of ILD activity and/or severity.

Renal crisis was defined as any two of the following three criteria: new-onset severe hypertension $(\geq 180 \mathrm{mmHg}$ systolic and/or $\geq 100 \mathrm{mmHg}$ diastolic) without an alternate aetiology, microangiopathic haemolytic anaemia, or rising creatinine.

SSc treatment was recorded at each visit as follows: corticosteroids (oral prednisolone), immunosuppressives (leflunomide, methotrexate, azathioprine, penicillamine, hydroxychloroquine, mycophenolate, cyclophosphamide and calcineurin inhibitors), biologic therapies (tumour necrosis factor- $\alpha$ inhibitors, tocilizumab, abatacept, anti-CD20 antibodies and other B-cell modulators) and home oxygen.

\section{Definition of SSc overlap syndrome}

The presence of 'overlap disease' was recorded at each annual visit and was defined as evidence of SSc together with characteristic symptoms or signs of overlap disease, which included persistent sicca symptoms, episodes of inflammatory myositis (muscle weakness together with two or more of elevated serum creatinine kinase, characteristic changes on electromyography and/or magnetic resonance imaging, or muscle biopsy showing typical histopathological changes), or a rheumatologist's report 
of overlap with another rheumatic disease, such as rheumatoid arthritis, polymyositis, dermatomyositis, SLE or Sjögren's syndrome.

\section{European Scleroderma Study Group disease activity score} The EScSG disease activity score and the presence of its ten components were recorded at each visit: scleroderma; digital necrosis; arthritis; MRSS $>14$; DLCO $<80 \%$ predicted; ESR >30 mm/h; hypocomplementaemia (low C3 and/or C4); and patient-reported changes in cardiopulmonary, vascular or skin symptoms in the preceding month. One point is assigned for the presence of each feature, and scores can range from 0 to 10 . However, because hypocomplementaemia is one of the ten variables in the EScSG activity score, we calculated a score based on the other nine variables only, in order to avoid overcorrelation in subsequent analysis. Therefore, final scores ranged from 0 to 9 , with higher scores indicating higher disease activity.

\section{Physician global assessment}

Physician global assessments of overall health, disease activity and disease damage were also recorded prospectively at each annual visit using a visual analogue scale. Physician global assessments entail the physician's taking note of all the available clinical and laboratory information in order to assign a score that ranges between 0 and 10 for each of overall health, disease activity and disease-related damage. Higher scores represent worse overall health, higher disease activity or greater diseaserelated damage.

\section{Statistical analysis}

Descriptive statistics (mean $\pm \mathrm{SD}$, median [interquartile range], minimum and maximum, and number [percent]) were used to describe the characteristics of the patients and the data set. As there was $<5 \%$ missing data for any particular variable, we did not impute missing data.

Univariable comparisons between patients who were persistently normocomplementaemic during follow-up and patients who had experienced at least one episode of hypocomplementaemia during the course of the study were made using the $t$ test for continuous variables, the chi-square test for categorical variables and the Wilcoxon signed-rank test for ordinal variables. In this analysis, disease manifestations were defined as 'present ever'. Additional analyses were performed comparing the two groups described above (persistent normocomplementaemia and at least one episode of hypocomplementaemia) with those who had persistent hypocomplementaemia at every visit during follow-up.

In multivariable analyses, the association between disease features at each visit and hypocomplementaemia at that particular visit was analysed. Here, we used generalised estimating equations (GEEs) to account for the expected correlation that arises when repeated measurements are taken from the same individual at multiple visits over time [21]. Another advantage of this method of analysis is that it overcomes any potential misclassification error that might arise from dividing patients into subgroups based on arbitrary definitions such as hypocomplementaemia ever, persistent hypocomplementaemia and persistent normocomplementaemia. Furthermore, as there is currently no consensus regarding meaningful changes in clinical parameters from one visit to the next, the use of GEEs enables evaluation of the relationship between hypocomplementaemia at a particular visit and actual clinical parameters at that particular visit.

All statistical analyses were performed using STATA 14 software (StataCorp, College Station, TX, USA). All $p$ values were two-tailed, and statistical significance was defined as $p \leq 0.05$. The overall study design and planned analyses are presented in Fig. 1.

\section{Results}

This study included 1140 patients with SSc who had complement levels recorded prospectively at one or more visits.

\section{Characteristics of cohort and data set}

The cohort presented in this study is summarized in Tables 1 and 2. Among the 1140 patients included in this study, the average age of patients was $46.0 \pm 14.1$ years at SSc diagnosis and $57.4 \pm 12.3$ years at recruitment, with a mean follow-up time of $3.4 \pm 1.7$ years. In terms of demographics, $87.2 \%$ of patients were female, $93.3 \%$ were white, $4.7 \%$ were Asian, $1.3 \%$ were Australian Aboriginal or Torres Strait Islander and $0.9 \%$ were of other racial origin. The mean disease duration at recruitment was $11.3 \pm$ 9.9 years, with $14.3 \%$ of patients having a disease duration $\leq 2$ years and $31.2 \%$ of patients having a disease duration $\leq 5$ years at recruitment. Diffuse SSc was present in $27.2 \%$ of patients, and $72.8 \%$ of patients had limited SSc.

SSc overlap disease features were present in $23.4 \%$ of patients. The autoantibody profile of patients is summarized in Table 1. Autoantibodies that were found in a significant proportion of the cohort included anti-Scl-70 (15.2\%), anti-centromere (46.2\%), anti-RNA polymerase (13.3\%), anti-dsDNA antibodies (13.3\%), ANCA (12.9\%), rheumatoid factor $(27.2 \%)$, anti-phospholipid $(25.7 \%)$ and anti- $\beta_{2}$-glycoprotein (33.0\%) antibodies.

Characteristics of the data set pertaining to complement measurements are summarized in Table 3. Hypocomplementaemia was present at recruitment in $13.2 \%$ of patients and thereafter present ever from recruitment to most recent visit in $24.1 \%$ of patients. The median number of complement measurements per patient was 2 , 


\section{Recruitment}

1140 SSc patients were recruited into the Australian Scleroderma Cohort Study (ASCS)

Demographic data, disease subtype and autoantibodies collected prospectively at recruitment

\section{Annual reviews}

Complement levels recorded prospectively at each annual visits

Diease manifestations, EScGS disease activity score and physician global assessments collected prospectively at each annual visit

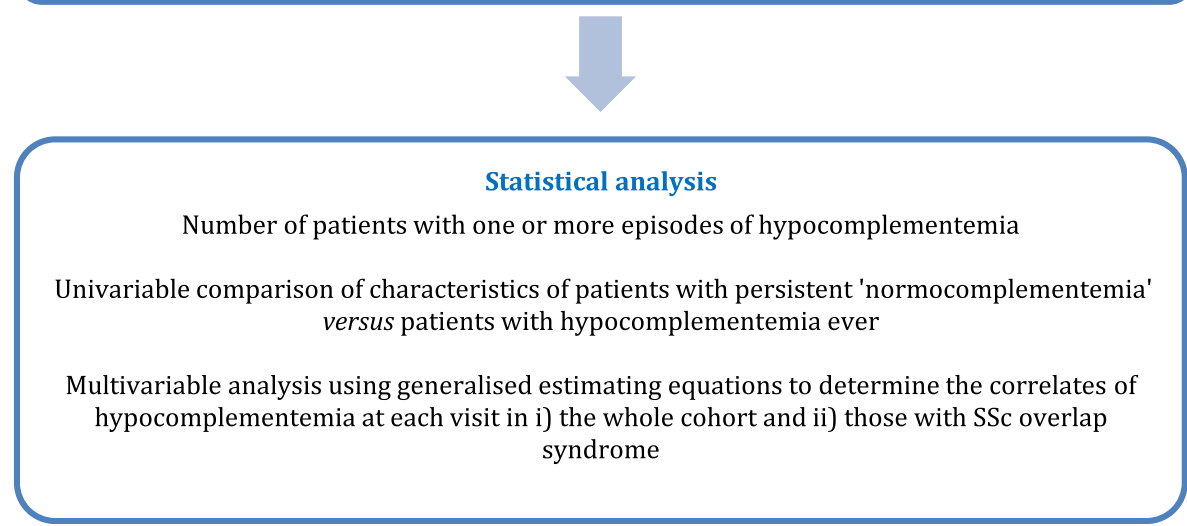

Fig. 1 Study design. ESCSG European Scleroderma Study Group, SSC Systemic sclerosis

with an interquartile range of $1-3$. The minimum number of measurements was 1 , and the maximum number of measurements was 8 . The average time interval between measurements was $1.2 \pm 0.4$ years. A total of 1893 individual visit measurements of complement with corresponding clinical and serological data were available for analysis.

\section{Univariable analyses}

Univariable comparisons between patients who had persistent normocomplementaemia during follow-up and patients who had experienced at least one episode of hypocomplementaemia during the course of the study are summarized in Tables 4 and 5. Patients who had ever been hypocomplementaemic were more likely to have features of overlap disease present $(29.5 \%$ vs. $21.5 \%$, $p=0.007)$, in particular polymyositis $(1.8 \%$ vs. $0.1 \%$, $p=0.001$ ). Hypocomplementaemia was also found to be associated with a number of autoantibodies typically associated with other rheumatic diseases, which included anti-RNP ( $4.5 \%$ vs. $1.2 \%, p=0.001)$, anti-Ro $(10.8 \%$ vs. $5.2 \%, p=0.001)$, anti-Sm (1.5\% vs. $0.2 \%, p=0.016)$ and anti-phospholipid (31.3 \% vs. $24.0 \%, p=0.02$ ) antibodies. Patients with hypocomplementaemia were also more likely to have a BMI $<20 \mathrm{~kg} / \mathrm{m}^{2}$ (20.5 \% vs. $13.0 \%, p=$ $0.003)$, a feature of more active and severe gastrointestinal involvement; scleroderma (72.8 \% vs. $66.2 \%, p=0.044)$; and muscle atrophy (23.2 \% vs $17.2 \%, p=0.029)$. A significant association with pericardial effusions $(9.1 \%$ vs. $5.3 \%, p=0.023$ ) was also found.

There was no difference in the mean EScSG disease activity score or physician global assessment score for overall health, disease activity and damage across all visits in patients who were normocomplementaemic compared with those who were hypocomplementaemic. In additional analyses, we found no significant differences between those who had at least one episode of hypocomplementaemia and persistent hypocomplementaemia during follow-up, possibly due to very few patients in the latter group (data not shown).

\section{Multivariable regression analyses}

In the analyses described above, variables were defined as being ever present from disease onset to most recent review. We performed a second series of analyses using GEEs with data gathered at each visit. This set comprised a total of 1893 visits. 
Table 1 Patient characteristics $(n=1140)$

\begin{tabular}{|c|c|}
\hline Characteristic & $n(\%)$ or mean $\pm \mathrm{SD}$ \\
\hline Age at diagnosis, years & $46.0 \pm 14.1$ \\
\hline Age at recruitment, years & $57.4 \pm 12.3$ \\
\hline Follow-up duration, years & $3.4 \pm 1.7$ \\
\hline \multicolumn{2}{|l|}{ Sex } \\
\hline Male & $148(13.0 \%)$ \\
\hline Female & $992(87.2 \%)$ \\
\hline \multicolumn{2}{|l|}{ Race } \\
\hline White & 1012 (93.3 \%) \\
\hline Asian & $50(4.7 \%)$ \\
\hline Australian Aboriginal or Torres Strait Islander & $14(1.3 \%)$ \\
\hline Other & $9(0.9 \%)$ \\
\hline Disease duration at recruitment ${ }^{a}$ & $11.3 \pm 9.9$ \\
\hline$\leq 2$ years & $163(14.3 \%)$ \\
\hline$\leq 5$ years & $356(31.2 \%)$ \\
\hline \multicolumn{2}{|l|}{ Disease subtype ${ }^{b}$} \\
\hline Diffuse & $310(27.2 \%)$ \\
\hline Limited & $830(72.8 \%)$ \\
\hline Overlap disease features present ever ${ }^{c}$ & $267(23.4 \%)$ \\
\hline Rheumatoid arthritis & $22(1.9 \%)$ \\
\hline Polymyositis & $6(0.5 \%)$ \\
\hline Dermatomyositis & $1(0.01 \%)$ \\
\hline Sjögren's syndrome & $21(1.8 \%)$ \\
\hline SLE & $9(0.8 \%)$ \\
\hline \multicolumn{2}{|l|}{ Serological profile at recruitment } \\
\hline Anti-centromere ANA & $517(46.2 \%)$ \\
\hline Anti-Scl-70 antibodies & $167(15.2 \%)$ \\
\hline Anti-RNAP antibodies & $84(13.3 \%)$ \\
\hline Anti-U1 RNP antibodies & $22(2.0 \%)$ \\
\hline Anti-Ro antibodies & $72(6.6 \%)$ \\
\hline Anti-La antibodies & $18(1.6 \%)$ \\
\hline Anti-Sm antibodies & $6(0.6 \%)$ \\
\hline Anti-PM-SCl antibodies & $15(1.4 \%)$ \\
\hline Anti-dsDNA antibodies & $26(3.1 \%)$ \\
\hline Anti-Jo-1 antibodies & $5(0.5 \%)$ \\
\hline ANCA & $134(12.9 \%)$ \\
\hline MPO specificity & $17(1.6 \%)$ \\
\hline PR-3 specificity & $22(2.1 \%)$ \\
\hline Rheumatoid factor & $288(27.2 \%)$ \\
\hline Anti-phospholipid antibodies & $272(25.7 \%)$ \\
\hline Cardiolipin IgM & $165(65.0 \%)$ \\
\hline Cardiolipin IgG & $105(39.3 \%)$ \\
\hline Anti- $\beta_{2}-$ glycoprotein antibody & $84(33.0 \%)$ \\
\hline Lupus anticoagulant & $26(3.1 \%)$ \\
\hline
\end{tabular}

Abbreviations: ANA Anti-nuclear antibody, Anti-Scl-70 Anti-scleroderma-70 antibodies, Anti-U1 RNP Anti-ribonucleoprotein antibodies, Anti-Sm Anti-Smith antibodies, AntiPM-Scl Anti-polymyositis scleroderma antibodies, Anti-dsDNA Anti-double-stranded DNA antibodies, Anti-RNAP Anti-RNA polymerase antibodies, ANCA Anti-neutrophil cytoplasmic antibodies, MPO Myeloperoxidase, PR-3 Proteinase-3

a Since onset of first non-Raynaud's phenomenon disease manifestation ${ }^{b}$ Disease subtype based on extent of skin involvement, with limited disease being confined to the extremities distal to elbows and knees, as well as the face ${ }^{c}$ Actual overlap disease features specified for only a proportion of patients classified by the treating physician as having 'SSc overlap syndrome'
We analysed data for up to six reviews per patient. We specified an exchangeable working correlation structure to account for the within-individual correlation and computed robust standard errors on the parametric estimates. We ran a univariable model of EScSG disease activity score (with the complement component removed to avoid over-correlation) and a multivariable model containing all of the individual disease activity variables listed in the EScSG disease activity index, with the exception of the complement item, and with some additional disease activity variables (C-reactive protein [CRP] $>8 \mathrm{mg} / \mathrm{L}$ and $\mathrm{BMI}$ ) included on the basis of univariable analyses (Table 6). Because scleroderma, muscle atrophy and pericardial effusion, which were statistically significant in univariable analysis, were not statistically significant in multivariable analysis, these variables were removed. Despite lack of significance in a simple univariable comparison, in a multivariable GEE model, FVC $<80 \%$ predicted was statistically significant and therefore included in the final model. We ran the multivariable models for (1) the whole cohort $(n=886 ; 1893$ visits in total) as well as (2) patients classified as having SSc overlap ( $n=221 ; 628$ visits in total) and (3) those with non-SSc overlap $(n=665 ; 1265$ visits in total) (Table 6).

In the multivariable analysis using the entire cohort, significant correlates of hypocomplementaemia at each visit included a lower $\mathrm{BMI}(\mathrm{OR}$ for $\mathrm{BMI}=0.90,95 \% \mathrm{CI}$ 0.88-0.94, $p<0.0005$ ), a lower MRSS (OR for MRSS = $0.98,95 \%$ CI $0.96-0.99, p=0.013$ ) and a lower ESR (OR for $\mathrm{ESR}=0.99,95 \%$ CI $0.98-0.99, p=0.022)$. In patients with SSc overlap disease features, significant correlates of hypocomplementaemia at each visit included digital ulcers $(\mathrm{OR}=1.62,95 \% \mathrm{CI} 1.04-2.51, p=0.034)$, tendon friction rubs $(\mathrm{OR}=2.31,95 \% \mathrm{CI} 1.05-5.10, p=0.037)$, an $\mathrm{FVC}<80 \%$ predicted (OR $=2.90,95 \%$ CI 1.32-6.38, $p=0.008)$ and a lower BMI (OR for BMI $=0.90,95 \% \mathrm{CI}$ $0.85-0.95, p<0.0005)$, all variables that are associated with increased SSc disease activity and/or severity. In patients without overlap disease features, significant correlates of hypocomplementaemia at each visit included a lower BMI (OR for BMI $=0.91,95 \%$ CI 0.87-0.95, $p<0.0005$ ) and a lower ESR (OR for ESR $=0.99,95 \%$ CI $0.97-0.99, p=0.026$ ).

\section{Discussion}

In this longitudinal cohort study of 1140 patients with SSc, we found that low serum complement occurred at least once in $23.4 \%$ of patients over the study period. We also found that these patients were more likely to have SSc overlap disease features than patients who were normocomplementaemic ( $29.5 \%$ vs. $21.5 \%, p=0.007)$, in particular polymyositis. We have also demonstrated that hypocomplementaemia is not a measure of disease 
Table 2 Disease manifestations in cohort $(n=1140)$

\begin{tabular}{|c|c|}
\hline Characteristics & $n(\%)$ or medi \\
\hline \multicolumn{2}{|l|}{ Disease manifestation ${ }^{a}$} \\
\hline Raynaud's phenomenon & 1065 (94.0\%) \\
\hline Digital ulcers & $345(30.5 \%)$ \\
\hline Digital gangrene/amputation & 93 (8.2 \%) \\
\hline Telangiectasia & $947(83.8 \%)$ \\
\hline Calcinosis & $439(38.9 \%)$ \\
\hline Scleroderma & $763(67.8 \%)$ \\
\hline Tendon friction rub & $112(10.0 \%)$ \\
\hline Joint contracture & $431(38.3 \%)$ \\
\hline Synovitis & $323(28.7 \%)$ \\
\hline Muscle atrophy & $210(18.7 \%)$ \\
\hline MRSS score $>20$ & $207(18.5 \%)$ \\
\hline Myocardial disease & $87(7.6 \%)$ \\
\hline Pericardial effusion ${ }^{b}$ & $70(6.2 \%)$ \\
\hline $\mathrm{PAH}^{\mathrm{C}}$ & $298(26.9 \%)$ \\
\hline $\operatorname{ILD}^{\mathrm{d}}$ & $340(30.1 \%)$ \\
\hline Gastrointestinal involvement ${ }^{\mathrm{e}}$ & $638(56.3 \%)$ \\
\hline GAVE & $113(10.0 \%)$ \\
\hline Reflux oesophagitis & $958(84.3 \%)$ \\
\hline Oesophageal stricture & $207(18.3 \%)$ \\
\hline Oesophageal dysmotility & $457(40.6 \%)$ \\
\hline Bowel dysmotility & $297(26.3 \%)$ \\
\hline Pseudo-obstruction & $37(3.3 \%)$ \\
\hline Renal crises ${ }^{f}$ & $44(3.9 \%)$ \\
\hline eGFR $<60 \mathrm{ml} /$ minute & $297(26.4 \%)$ \\
\hline Myositis $^{9}$ & $58(6.0 \%)$ \\
\hline $\mathrm{CRP}>8 \mathrm{mg} / \mathrm{L}$ & $333(29.7 \%)$ \\
\hline $\mathrm{ESR}>30 \mathrm{~mm} / \mathrm{h}$ & $323(28.8 \%)$ \\
\hline Blood CK > 200 IU/L & $122(11.0 \%)$ \\
\hline Anaemia $^{\text {h }}$ & $403(35.5 \%)$ \\
\hline FVC $<80 \%$ predicted & $298(26.9 \%)$ \\
\hline DLCO <80\% predicted & $832(79.9 \%)$ \\
\hline EScSG disease activity score ${ }^{i}$ & $2.5(1-4)$ \\
\hline \multicolumn{2}{|l|}{ Treatments $^{a}$} \\
\hline Corticosteroids & $506(44.4 \%)$ \\
\hline Immunotherapy ${ }^{j}$ & $495(43.4 \%)$ \\
\hline Biological therapyk & $16(1.4 \%)$ \\
\hline Home oxygen & $41(3.6 \%)$ \\
\hline
\end{tabular}

Table 2 Disease manifestations in cohort ( $n=1140)$ (Continued)

\begin{tabular}{ll}
\hline Physician global assessments $^{\prime}$ & \\
Overall health $^{\mathrm{m}}$ & $4(3-6)$ \\
Activity $^{\mathrm{n}}$ & $3(2-5)$ \\
Damage $^{\mathrm{n}}$ & $4(3-6)$
\end{tabular}

Abbreviations: MRSS Modified Rodnan skin score, ESCSG European Scleroderma Study Group, PAH Pulmonary arterial hypertension, ILD Interstitial lung disease, GAVE Gastric antral vascular ectasia, eGFR Estimated glomerular filtration rate, $C R P$ C-reactive protein, ESR Erythrocyte sedimentation rate, CK Creatinine kinase, FVC Forced vital capacity, DLCO Diffusing capacity of the lung for carbon monoxide corrected for haemoglobin

${ }^{a}$ Ever from disease onset to most recent visit

bericardial effusion defined by echocardiography

CPAH defined by right heart catheterization with a mean pulmonary artery pressure $\geq 25 \mathrm{mmHg}$ and a pulmonary arterial wedge pressure $\leq 15 \mathrm{mmHg}$

dPulmonary fibrosis defined by chest high-resolution computed tomography e'Gastrointestinal symptoms defined as the presence of any of reflux,

dysphagia, post-prandial bloating, vomiting, constipation, diarrhoea or anal incontinence as defined in text

${ }^{f}$ Renal crisis defined as the presence of at least two of the following: new-onset hypertension, rising creatinine or microangiopathic haemolytic anaemia ${ }^{9}$ Myositis defined as either definite (biopsy), suspected (CK or electromyogram) or possible (magnetic resonance imaging scan)

${ }^{\mathrm{h}}$ Anaemia defined as haemoglobin level $<135 \mathrm{~g} / \mathrm{L}$ in males and $<120 \mathrm{~g} / \mathrm{L}$ in females

'Calculated without hypocomplementaemia. Final scores range from 0 to 9, with higher scores indicating higher disease activity

Includes leflunomide, methotrexate, azathioprine, penicillamine, hydroxychloroquine, mycophenolate, cyclophosphamide and calcineurin inhibitors

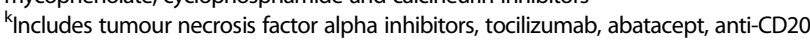
antibodies and other B-cell modulators

'Highest score ever recorded over the study period

${ }^{\mathrm{m}} \mathrm{Scores}$ range from 0 to 10 , with higher scores being indicative of worse overall health

${ }^{\mathrm{n}}$ Scores range from 0 to 10 , with higher scores being indicative of higher disease activity or damage

Table 3 Characterisation of complement levels $(n=1140)$

\begin{tabular}{|c|c|}
\hline Parameter & $\begin{array}{l}n(\%) \text { or mean } \pm \text { SD or median } \\
\text { (25th-75th IQR) }\end{array}$ \\
\hline \multicolumn{2}{|l|}{ Hypocomplementaemia ${ }^{a}$} \\
\hline At recruitment & $150(13.2 \%)$ \\
\hline Ever $^{b}$ & 275 (24.1\%) \\
\hline $\begin{array}{l}\text { Number of complement } \\
\text { measurements per patient }\end{array}$ & $2(1-3)$ \\
\hline 1 & $400(35.1 \%)$ \\
\hline 2 & 265 (23.3 \%) \\
\hline 3 & 196 (17.2 \%) \\
\hline 4 & $129(11.3 \%)$ \\
\hline 5 & 85 (7.5 \%) \\
\hline 6 & $50(4.4 \%)$ \\
\hline 7 & $14(1.2 \%)$ \\
\hline 8 & 1 (0.1\%) \\
\hline $\begin{array}{l}\text { Total number of complement } \\
\text { measurements in data set }\end{array}$ & 1893 \\
\hline $\begin{array}{l}\text { Time interval between complement } \\
\text { measurements, years }\end{array}$ & $1.2 \pm 0.4$ \\
\hline
\end{tabular}

${ }^{\mathrm{a} A}$ low $\mathrm{C} 3$ and/or $\mathrm{C} 4$ result

${ }^{\mathrm{b}}$ At least one episode of hypocomplementaemia over the study period 
Table 4 Univariable comparison of demographics, disease subtypes and serological profiles in patients with persistent normocomplementaemia and those with at least one episode of hypocomplementaemia

\begin{tabular}{|c|c|c|c|}
\hline Characteristic & Persistent normocomplementaemia $(n=865)$ & At least one episode of hypocomplementaemia $(n=275)$ & \\
\hline & $n(\%)$ or mean $\pm \mathrm{SD}$ or median (IQR) & $n(\%)$ or mean $\pm \mathrm{SD}$ or median (IQR) & $p$ Value \\
\hline Age at diagnosis, years & $46.4 \pm 0.5$ & $44.6 \pm 0.8$ & 0.071 \\
\hline \multicolumn{4}{|l|}{ Sex } \\
\hline Male & $113(13.1 \%)$ & 35 (12.7\%) & 0.89 \\
\hline Female & $752(86.9 \%)$ & $240(87.3 \%)$ & 0.89 \\
\hline Disease duration & $11.3 \pm 0.4$ & $11.6 \pm 0.7$ & 0.71 \\
\hline$\leq 2$ years & $117(14.5 \%)$ & $46(17.5 \%)$ & 0.24 \\
\hline$\leq 5$ years & $268(33.2 \%)$ & $88(33.5 \%)$ & 0.94 \\
\hline \multicolumn{4}{|l|}{ Disease type } \\
\hline Diffuse & $238(27.5 \%)$ & $72(26.2 \%)$ & 0.67 \\
\hline Limited & $627(72.5 \%)$ & $203(73.8 \%)$ & 0.67 \\
\hline Overlap features present ${ }^{\mathrm{a}}$ & $186(21.5 \%)$ & 81 (29.5\%) & 0.007 \\
\hline Rheumatoid arthritis & $15(1.7 \%)$ & $7(2.6 \%)$ & 0.39 \\
\hline Polymyositis & $1(0.1 \%)$ & $5(1.8 \%)$ & 0.001 \\
\hline Dermatomyositis & $0(0 \%)$ & $1(0.4 \%)$ & 0.076 \\
\hline Sjögren's syndrome & $14(1.6 \%)$ & $7(2.6 \%)$ & 0.32 \\
\hline SLE & $6(0.7 \%)$ & $3(1.1 \%)$ & 0.52 \\
\hline \multicolumn{4}{|l|}{ Serological profile ${ }^{a}$} \\
\hline Anti-centromere ANA & 391 (46.0 \%) & $126(47.0 \%)$ & 0.084 \\
\hline Anti-Scl-70 antibodies & $134(16.1 \%)$ & $33(12.3 \%)$ & 0.13 \\
\hline Anti-Jo-1 antibodies & $4(0.5 \%)$ & $1(0.4 \%)$ & 0.82 \\
\hline Anti-RNP antibodies & $10(1.2 \%)$ & $12(4.5 \%)$ & 0.001 \\
\hline Anti-Ro antibodies & $43(5.2 \%)$ & $29(10.8 \%)$ & 0.001 \\
\hline Anti-La antibodies & $12(1.5 \%)$ & $6(2.2 \%)$ & 0.38 \\
\hline Anti-Sm antibodies & $2(0.2 \%)$ & $4(1.5 \%)$ & 0.016 \\
\hline Anti-PM-SCl antibodies & $10(1.2 \%)$ & $5(1.9 \%)$ & 0.43 \\
\hline Anti-dsDNA antibodies & $16(2.5 \%)$ & $10(4.9 \%)$ & 0.089 \\
\hline Anti-RNAP antibodies & $61(13.6 \%)$ & $23(12.6 \%)$ & 0.74 \\
\hline ANCA & 95 (12.1\%) & 39 (15.2\%) & 0.20 \\
\hline MPO specificity & $14(1.8 \%)$ & $3(1.2 \%)$ & 0.50 \\
\hline PR-3 specificity & $14(1.8 \%)$ & $8(3.1 \%)$ & 0.20 \\
\hline Rheumatoid factor & $208(26.1 \%)$ & $80(31.3 \%)$ & 0.11 \\
\hline Anti-phospholipid antibodies & $192(24.0 \%)$ & $80(31.3 \%)$ & 0.02 \\
\hline Cardiolipin IgM & $115(63.2 \%)$ & $50(69.4 \%)$ & 0.351 \\
\hline Cardiolipin IgG & $78(41.5 \%)$ & $27(34.2 \%)$ & 0.26 \\
\hline$\beta_{2}$-glycoprotein & $59(32.8 \%)$ & 25 (33.3 \%) & 0.93 \\
\hline Lupus anti-coagulant & $15(2.4 \%)$ & $11(5.0 \%)$ & 0.056 \\
\hline
\end{tabular}

Abbreviations: ANA Anti-nuclear antibody, Anti-Scl-70 Anti-scleroderma-70 antibodies, Anti-RNP Anti-ribonucleoprotein antibodies, Anti-Sm Anti-Smith antibodies, Anti-PM-Scl Anti-polymyositis scleroderma antibodies, Anti-dsDNA Anti-double-stranded DNA antibodies, Anti-RNAP Anti-RNA polymerase antibodies, ANCA Anti-neutrophil cytoplasmic antibodies, MPO Myeloperoxidase, PR-3 Proteinase-3, lg Immunoglobulin, SLE Systemic lupus erythematosus

${ }^{a}$ Ever from disease onset to most recent visit

activity when applied across the entire cohort of patients with SSc. However, to our knowledge, this is the first study to demonstrate that hypocomplementaemia is a marker of the presence of certain SSc disease features measured at each visit among the subset of patients with overlap disease features. These disease features include digital ulcers, tendon friction rubs, low BMI and low FVC, all of which are clinical features of SSc disease 
Table 5 Univariable associations of hypocomplementaemia with clinical manifestations, treatment, European Scleroderma Study Group disease activity score and physician global assessments

\begin{tabular}{|c|c|c|c|}
\hline \multirow[t]{2}{*}{ Characteristic } & \multirow{2}{*}{$\begin{array}{l}\text { Persistent normocomplementaemia }(n=859) \\
n(\%) \text { or mean } \pm \text { SD or median (IQR) }\end{array}$} & \multicolumn{2}{|l|}{ At least one episode of hypocomplementaemia $(n=275)$} \\
\hline & & $n(\%)$ or mean \pm SD or median (IQR) & $p$ Value \\
\hline \multicolumn{4}{|l|}{ Disease manifestation $^{a}$} \\
\hline Raynaud's phenomenon & $803(93.5 \%)$ & $262(95.6 \%)$ & 0.19 \\
\hline $\mathrm{BMI}<20 \mathrm{~kg} / \mathrm{m}^{2}$ & $104(13.0 \%)$ & $54(20.5 \%)$ & 0.0030 \\
\hline Digital ulcers & $263(30.7 \%)$ & $82(30.0 \%)$ & 0.848 \\
\hline Digital gangrene/amputation & $76(8.8 \%)$ & $17(6.2 \%)$ & 0.17 \\
\hline Telangiectasia & $716(83.6 \%)$ & $231(84.6 \%)$ & 0.68 \\
\hline Calcinosis & $340(39.7 \%)$ & $99(36.3 \%)$ & 0.31 \\
\hline Scleroderma & $565(66.2 \%)$ & $198(72.8 \%)$ & 0.044 \\
\hline Tendon friction rub & $88(10.3 \%)$ & $24(8.8 \%)$ & 0.47 \\
\hline Joint contracture & $332(38.9 \%)$ & 99 (36.4 \%) & 0.46 \\
\hline Synovitis & $241(28.3 \%)$ & $82(30.0 \%)$ & 0.59 \\
\hline Muscle atrophy & $147(17.2 \%)$ & $63(23.2 \%)$ & 0.029 \\
\hline MRSS > 20 & $158(18.7 \%)$ & 49 (18.0\%) & 0.81 \\
\hline Myocardial disease & 70 (8.1\%) & $17(6.2 \%)$ & 0.30 \\
\hline Pericardial effusion & $45(5.3 \%)$ & $25(9.1 \%)$ & 0.023 \\
\hline PAH & $90(10.4 \%)$ & 30 (10.9\%) & 0.82 \\
\hline Pulmonary fibrosis & $263(30.8 \%)$ & 77 (30.1 \%) & 0.40 \\
\hline Gastrointestinal involvement & $493(57.5 \%)$ & $145(52.7 \%)$ & 0.17 \\
\hline GAVE & $86(10.1 \%)$ & $27(9.9 \%)$ & 0.94 \\
\hline Reflux oesophagitis & $722(83.8 \%)$ & $236(85.8 \%)$ & 0.41 \\
\hline Oesophageal stricture & $109(12.8 \%)$ & $23(8.4 \%)$ & 0.051 \\
\hline Oesophageal dysmotility & $346(40.5 \%)$ & $111(40.8 \%)$ & 0.92 \\
\hline Bowel dysmotility & $226(26.4 \%)$ & $71(25.9 \%)$ & 0.88 \\
\hline Pseudo-obstruction & $25(2.9 \%)$ & $12(4.4 \%)$ & 0.23 \\
\hline Renal crises & $36(4.2 \%)$ & $8(2.9 \%)$ & 0.34 \\
\hline eGFR $<60 \mathrm{ml} /$ minute & $229(22.8 \%)$ & $68(25.0 \%)$ & 0.55 \\
\hline Myositis & $37(5.2 \%)$ & $21(8.5 \%)$ & 0.055 \\
\hline $\mathrm{CRP}>8 \mathrm{mg} / \mathrm{L}$ & 266 (31.1 \%) & 67 (24.9\%) & 0.051 \\
\hline $\mathrm{ESR}>30 \mathrm{~mm} / \mathrm{h}$ & 252 (29.6 \%) & 71 (26.1\%) & 0.27 \\
\hline Blood CK > 200 IU/L & 105 (12.1\%) & 44 (16.0\%) & 0.098 \\
\hline Anaemia & 302 (35.1 \%) & $101(36.7 \%)$ & 0.62 \\
\hline FVC $<80 \%$ & 225 (26.9 \%) & 73 (27.0 \%) & 0.97 \\
\hline DLCO <80 \% & 631 (80.5 \%) & 201 (78.2 \%) & 0.43 \\
\hline \multicolumn{4}{|l|}{ Treatment ${ }^{a}$} \\
\hline Corticosteroids & $380(43.9 \%)$ & $126(45.8 \%)$ & 0.58 \\
\hline Immunotherapy & 364 (42.1 \%) & 131 (47.6 \%) & 0.11 \\
\hline Biologic therapy & $9(1.0 \%)$ & 7 (2.6 \%) & 0.065 \\
\hline Home oxygen & $32(3.7 \%)$ & $9(3.3 \%)$ & 0.74 \\
\hline EScSG score & $2.5(1.4)$ & $2(1-4)$ & 0.16 \\
\hline \multicolumn{4}{|l|}{ Physician global assessments ${ }^{b}$} \\
\hline Health & $4(3-6)$ & $4(3-6)$ & 0.25 \\
\hline Activity & $3(2-5)$ & $3(2-5)$ & 0.076 \\
\hline Damage & $4(2.5-6)$ & $4(3-6)$ & 0.44 \\
\hline
\end{tabular}

Abbreviations: MRSS Modified Rodnan skin score, ESCSG European Scleroderma Study Group, PAH Pulmonary arterial hypertension, ILD Interstitial lung disease, GAVE Gastric antral vascular ectasia, BMI Body mass index, eGFR Estimated glomerular filtration rate, CRP C-reactive protein, ESR Erythrocyte sedimentation rate, CK Creatinine kinase, FVC Forced vital capacity, DLCO Diffusing capacity of the lung for carbon monoxide corrected for haemoglobin

${ }^{\mathrm{a}}$ Ever from disease onset to most recent visit

${ }^{\mathrm{b}}$ Mean score from all visits 
Table 6 Multivariable associations of hypocomplementaemia with features of disease activity at each visit among the whole cohort and analysis subsets determined using generalised estimating equations

\begin{tabular}{|c|c|c|c|c|c|c|}
\hline \multirow[b]{2}{*}{ Parameter } & \multicolumn{2}{|c|}{$\begin{array}{l}\text { Whole cohort } \\
\text { ( } n=886 ; 1893 \text { visits) }\end{array}$} & \multicolumn{2}{|c|}{$\begin{array}{l}\text { SSc with features of overlap } \\
\text { disease ( } n=221 ; 628 \text { visits) }\end{array}$} & \multicolumn{2}{|c|}{$\begin{array}{l}\text { SSc without features of overlap } \\
\text { disease ( } n=665 ; 1265 \text { visits) }\end{array}$} \\
\hline & $\begin{array}{l}\text { Odds ratio } \\
(95 \% \mathrm{Cl})\end{array}$ & $p$ Value & $\begin{array}{l}\text { Odds ratio } \\
(95 \% \mathrm{Cl})\end{array}$ & $p$ Value & $\begin{array}{l}\text { Odds ratio } \\
(95 \% \mathrm{Cl})\end{array}$ & $p$ Value \\
\hline $\begin{array}{l}\text { Self-reported worsening of cardiopulmonary, } \\
\text { vascular or skin symptoms }\end{array}$ & $1.05(0.57-1.94)$ & 0.87 & $1.24(0.48-3.18)$ & 0.66 & $1.03(0.45-2.35)$ & 0.94 \\
\hline Digital ulcers/necrosis ${ }^{\mathrm{a}}$ & $1.15(0.84-1.56)$ & 0.38 & $1.62(1.04-2.51)$ & 0.034 & $0.82(0.53-1.27)$ & 0.37 \\
\hline Scleroderma ${ }^{a}$ & $0.97(0.76-1.23)$ & 0.78 & $1.08(0.75-1.58)$ & 0.67 & $0.87(0.63-1.18)$ & 0.37 \\
\hline Tendon friction rubs ${ }^{a}$ & $1.15(0.64-2.08)$ & 0.64 & $2.31(1.05-5.10$ & 0.037 & $0.54(0.19-1.53)$ & 0.24 \\
\hline Synovitis/arthritis ${ }^{a}$ & $0.95(0.69-1.31)$ & 0.76 & $1.18(0.73-1.91)$ & 0.50 & $0.80(0.51-1.25)$ & 0.32 \\
\hline Modified Rodnan skin score $>14^{a}$ & $0.98(0.96-0.99)$ & 0.013 & $0.97(0.95-1.01)$ & 0.087 & $0.98(0.96-1.01)$ & 0.16 \\
\hline Erythrocyte sedimentation rate $>30 \mathrm{~mm} / \mathrm{h}^{\mathrm{a}}$ & $0.98(0.98-0.99)$ & 0.022 & $0.99(0.99-1.01)$ & 0.97 & $0.99(0.97-0.99)$ & 0.026 \\
\hline DLCO $<80 \%$ predicted $^{a}$ & $0.81(0.61-1.09)$ & 0.17 & $1.14(0.66-1.96)$ & 0.65 & $0.72(0.51-1.03)$ & 0.073 \\
\hline C-reactive protein $>8 \mathrm{mg} / \mathrm{L}^{\mathrm{a}}$ & $1.01(0.99-1.01)$ & 0.90 & $0.97(0.93-1.01)$ & 0.073 & $1.01(0.99-1.01)$ & 0.41 \\
\hline$B M I^{b}$ & $0.91(0.88-0.94)$ & $<0.0005$ & $0.90(0.85-0.95)$ & $<0.0005$ & $0.91(0.87-0.95)$ & $<0.0005$ \\
\hline FVC $<80 \%$ predicted $^{b}$ & $1.24(0.78-1.97)$ & 0.37 & $2.90(1.32-6.38)$ & 0.0080 & $0.87(0.47-1.61)$ & 0.65 \\
\hline
\end{tabular}

Abbreviations: BMI Body mass index, FVC forced vital capacity, DLCO Diffusing capacity of the lung for carbon monoxide corrected for haemoglobin

All variables listed in the table were included in the final multivariable generalised estimating equation model

a Components of European Scleroderma Study Group disease activity index

${ }^{\mathrm{b}}$ Additional disease activity variables entered into multivariable regression model on the basis of significance in univariable analysis

activity or severity. This in turn suggests a role for measurement of $\mathrm{C} 3$ and $\mathrm{C} 4$ in assessment and monitoring of disease activity in patients with overlap SSc.

We found that the prevalence of hypocomplementaemia at recruitment was $13.2 \%$, which is consistent with previous smaller studies $[22,23]$. The finding that hypocomplementaemia is associated with the presence of overlap disease features in SSc is also consistent with trends identified by Hudson et al. in a cohort of 321 Canadian patients with SSc [23]. This suggests that complement consumption plays a significant role in the etiopathogenesis of SSc overlap disease.

Several small studies have cited a role for abnormal complement activation in SSc. Senaldi et al. found that the levels of serum complement fragments $\mathrm{Ba}, \mathrm{C} 3 \mathrm{~d}$ and C4d were higher in patients with diffuse SSc than in patients with limited SSc and that both subtypes had serum complement fragment levels higher than those of normal patients, suggesting that complement consumption occurs in SSc and may also be related to disease activity and/or severity [24]. Batal et al. undertook a study of prognostic factors in systemic sclerosis renal crisis (SRC) in which they retrospectively analysed renal biopsies. They found that peritubular capillary C4d deposition was higher in patients with SRC than in both hypertensive non-SRC and normotensive nonSRC control subjects and suggested a role for immune complex- and antibody-mediated injury in SRC [25]. Whilst in vivo activation of the complement system in SSc remains a possibility, the general consensus is that complement consumption does not play a significant role in the pathophysiology of SSc and that therefore the presence of hypocomplementaemia in SSc signifies the presence of another overlapping disease. This is consistent with the results of our study, which demonstrated a significant association between hypocomplementaemia and the presence of SSc overlap disease, in particular polymyositis. However, it is intriguing to note that even among patients with overlap disease, low serum complement was associated with disease features classically associated with SSc itself, such as digital ulcers and tendon friction rubs.

Hypocomplementaemia is one of the ten variables listed in the EScSG disease activity index [26, 27]. However, its inclusion has been a point of controversy. In the present study, we were unable to demonstrate a consistent association between hypocomplementaemia and either the EScSG disease activity score (calculated on the basis of nine non-complement variables to avoid over-correlation between independent variables) or any of its component variables in the univariable analysis. In addition, the associations found in the multivariable analysis in the subset of patients with SSc without overlap disease features (non-overlap SSc) suggest that, compared with normocomplementaemic patients, hypocomplementaemic patients have lower EScSG disease activity scores and ESR levels, which is the opposite of what one might expect. This lack of expected correlation with SSc disease activity measures suggests that hypocomplementaemia is not a measure of disease activity in the majority of patients with SSc, which is consistent with the conclusions drawn from previous studies [23]. Only one study suggested the contrary. Cuomo et al. reported the associations between 
hypocomplementaemia and clinical status in a cohort of 302 Italian patients with SSc and excluded patients with overlap disease features. They found that $16.5 \%$ of their cohort had hypocomplementaemia and that hypocomplementaemia was associated with significantly increased disease activity, significantly increased severity of disease manifestations in the skin, cardiovascular and respiratory systems, and greater functional disability [22]. However, it is unclear whether the results of their study are generalizable to other SSc cohorts, because there was a very high prevalence of patients with anti-Scl-70 and anti-centromere ANA. The latter autoantibodies in particular have been suggested to play a role in complement activation [28].

The lack of association between hypocomplementaemia and other potential markers of disease activity in the EScSG disease activity index may also speak to the latter's not being a very good measure of 'disease activity' per se. A trend toward a significant difference in the physician global assessment of disease activity between the persistently normocomplementaemic and the hypocomplementaemic groups $(p=0.076)$ supports this statement. However, it is also possible that each item in the EScSG disease activity index measures disease activity independently of all other items. Furthermore, it must be noted that functional abnormalities in complement in SSc may occur in the absence of hypocomplementaemia.

To our knowledge, this is the first study to use GEE methods to definitively show that hypocomplementaemia is associated with some features of disease activity in patients with SSc who have overlap disease features. We found that within this subset of patients with SSc, those who were hypocomplementaemic were more likely to manifest digital ulcers, tendon friction rubs, an FVC $<80 \%$ predicted and a lower BMI at each visit, all of which are well-established features of disease activity and severity in non-overlap SSc itself. No association was found with either CRP or ESR, possibly because these inflammatory markers generally are not considered to be good measures of disease activity in SSc. In particular, it has been shown that CRP levels are not elevated in most patients with SSc and that CRP levels regress as disease duration increases [29].

This study is not without limitations. As patients had varying lengths of follow-up, some had more annual complement measurements than others, making it possible that there was a low complement reading by chance alone on one or more occasions. However, the use of GEEs for statistical analysis overcomes this issue to a degree because it enables hypocomplementaemia at each visit to be associated with disease features at that particular visit, eliminating the need to divide patients into subgroups based on arbitrary definitions such as hypocomplementaemia ever, persistent hypocomplementaemia and persistent normocomplementaemia.

\section{Conclusions}

In this study, we have shown that hypocomplementaemia in SSc is associated with the presence of SSc overlap features, in particular SSc-polymyositis overlap syndrome. We have also confirmed the results of previous studies by showing that hypocomplementaemia is not a measure of disease activity in non-overlap SSc cohorts. However, to our knowledge, this is the first study to show that hypocomplementaemia is associated with features of disease activity in SSc overlap disease and that, in these patients, the measurement of complement levels is potentially useful for monitoring disease activity and response to treatment.

\section{Abbreviations \\ ANA: Anti-nuclear antibodies; ANCA: Anti-neutrophil cytoplasmic antibodies; Anti-dsDNA: Anti-double-stranded DNA; Anti-PM-Scl: Anti-polymyositis scleroderma antibodies; Anti-RNAP: Anti-RNA polymerase antibodies; Anti-Scl-70: Anti-scleroderma-70 antibodies; Anti-Sm: Anti-Smith; Anti-U1 RNP: Anti-ribonucleoprotein antibodies; BMI: Body mass index; CK: Creatine kinase; CRP: C-reactive protein; DLCO: Diffusing capacity of the lung for carbon monoxide; eGFR: Estimated glomerular filtration rate; ELISA: Enzyme- linked immunosorbent assay; EScSG: European Scleroderma Study Group; ESR: Erythrocyte sedimentation rate; FVC: Forced vital capacity; GAVE: Gastric antral vascular ectasia; GEE: Generalised estimating equation; lg: Immunoglobulin; ILD: Interstitial lung disease; MPO: Myeloperoxidase; MRSS: Modified Rodnan skin score; PAH: Pulmonary arterial hypertension; PIIINP: Type III procollagen aminopeptide; PR-3: Proteinase-3 specificity; RNA Pol: RNA polymerase; RNP: Ribonucleoprotein; IIL-2R: Soluble interleukin-2 receptor; SLE: Systemic lupus erythematosus; SRC: Systemic sclerosis renal crisis; SSc: Systemic sclerosis; TLR: Toll-like receptor}

\section{Acknowledgements}

We thank Dr Peter Youssef, Dr Alan Sturgess, Dr Fiona Kermeen, Dr Catherine Hill and Dr Peter Nash for contributing data.

\section{Funding}

MN holds a National Health and Medical Research Council fellowship (APP1071735). This work was supported by Scleroderma Australia, Arthritis Australia, Actelion Pharmaceuticals Australia, Bayer, CSL Behring, GlaxoSmithKline Australia and Pfizer.

Availability of data and materials

Data sets on which the conclusions of this article rely have not been disclosed.

\section{Authors' contributions}

JE designed the study, analysed the data, interpreted the results and prepared the manuscript. WS designed the study, collected data, interpreted the results and prepared the manuscript. ZB, CR, JS, JZ, JR, JW and SMP collected data, interpreted the results and prepared the manuscript. MN designed the study, collected and analysed data, interpreted the results and prepared the manuscript. All authors read and approved the final manuscript.

\section{Competing interests}

The authors declare that they have no competing interests.

\section{Consent for publication \\ Not applicable.}

\section{Ethics approval and consent to participate}

The study was approved by the human research ethics committees of each of the participating centres. Consent to participate was obtained from all patients prior to the collection of demographic and disease-related data (including clinical and laboratory data) according to a standardized protocol. 


\section{Author details}

'Department of Medicine, The University of Melbourne at St Vincent's Hospital (Melbourne), 41 Victoria Parade, Fitzroy, VIC 3065, Australia. 2Department of Rheumatology, St Vincent's Hospital (Melbourne), 41 Victoria Parade, Fitzroy, VIC 3065, Australia. ${ }^{3}$ Department of Rheumatology, Monash Health and Monash University, 246 Clayton Road, Clayton, VIC 3168, Australia. ${ }^{4}$ Department of Medicine, Monash Health and Monash University, 246 Clayton Road, Clayton, VIC 3168, Australia. ${ }^{5}$ Department of Rheumatology, Menzies Institute for Medical Research, Private Bag 23, Hobart, TAS 7001, Australia. ${ }^{6}$ Department of Rheumatology, Royal Perth Hospital, 197 Wellington Street, GPO Box X2213, Perth, WA 6001, Australia. 'Department of Rheumatology, Flinders Medical Centre, Flinders Drive, Bedford Park, SA 5042, Australia. ${ }^{8}$ Rheumatology Unit, Royal Adelaide Hospital, North Terrace, Adelaide, SA 5000, Australia. 'Discipline of Medicine, University of Adelaide, Adelaide, SA 5000, Australia.

\section{Received: 29 June 2016 Accepted: 5 October 2016}

\section{Published online: 22 October 2016}

\section{References}

1. Nikpour M, Stevens WM, Herrick AL, Proudman SM. Epidemiology of systemic sclerosis. Best Pract Res Clin Rheumatol. 2010;24(6):857-69.

2. Gabrielli A, Avvedimento EV, Krieg T. Scleroderma. N Engl J Med. 2009; 360(19):1989-2003.

3. Medsger Jr TA. Natural history of systemic sclerosis and the assessment of disease activity, severity, functional status, and psychologic well-being Rheum Dis Clin North Am. 2003;29(2):255-73.

4. Symmons DPM. Disease assessment indexes: activity, damage and severity. Baillieres Clin Rheumatol. 1995;9(2):267-85.

5. Gladman DD, Ibañez D, Urowitz MB. Systemic Lupus Erythematosus Disease Activity Index 2000. J Rheumatol. 2002;29(2):288-91.

6. Valentini G, Silman AJ, Veale D. Assessment of disease activity. Clin Exp Rheumatol. 2003:21(3 Suppl 29):S39-41.

7. Della Rossa A, Valentini G, Bombardieri S, Bencivelli W, Silman AJ, D’Angelo $\mathrm{S}$, et al. European multicentre study to define disease activity criteria for systemic sclerosis. I. Clinical and epidemiological features of 290 patients from 19 centres. Ann Rheum Dis. 2001:60(6):585-91.

8. Valentini G, Della Rossa A, Bombardieri S, Bencivelli W, Silman AJ, D'Angelo S, et al. European multicentre study to define disease activity criteria for systemic sclerosis. II. Identification of disease activity variables and development of preliminary activity indexes. Ann Rheum Dis. 2001;60(6):592-8.

9. Scheja A, Akesson A, Horslev-Petersen K. Serum levels of aminoterminal type III procollagen peptide and hyaluronan predict mortality in systemic sclerosis. Scand J Rheumatol. 1992:21:5-9.

10. Steen VD, Engel EE, Charley MR, Medsger Jr TA. Soluble serum interleukin 2 receptors in patients with systemic sclerosis. J Rheumatol. 1996;23(4):646-9.

11. Vischer U, Ingerslev J, Wollheim C, Mestries JC, Tsakiris D, Haefeli W, et al. Acute von Willebrand factor secretion from the endothelium in vivo: assessment through plasma propeptide (vWf:Agll) levels. Thromb Haemost. 1997:77:387-93.

12. Clements PJ, Peter JB, Agopian MS, Telian NS, Furst DE. Elevated serum levels of soluble interleukin 2 receptor, interleukin 2 and neopterin in diffuse and limited scleroderma: effects of chlorambucil. J Rheumatol. 1990;17(7):908-10.

13. Stevens W, Vancheeswaran R. Black CM; UK Systemic Sclerosis Study Group. Alpha interferon-2a (Roferon-A) in the treatment of diffuse cutaneous systemic sclerosis: a pilot study. Br J Rheumatol. 1992;31(10):683-9.

14. Chen M, Daha MR, Kallenberg CGM. The complement system in systemic autoimmune disease. J Autoimmun. 2010:34(3):J276-86.

15. Valentijn RM, van Overhagen $H$, Hazevoet HM, Hermans J, Cats A, Daha MR, et al. The value of complement and immune complex determinations in monitoring disease activity in patients with systemic lupus erythematosus. Arthritis Rheum. 1985;28(8):904-13.

16. Sturfelt G, Sjoholm AG. Complement components, complement activation, and acute phase response in systemic lupus erythematosus. Arthritis Care Res (Hoboken). 1984;75(1):75-83.

17. Bhattacharyya S, Varga J. Emerging roles of innate immune signaling and Toll-like receptors in fibrosis and systemic sclerosis. Curr Rheumatol Rep. 2015;17(1):474

18. van den Hoogen F, Khanna D, Fransen J, Johnson SR, Baron M, Tyndall A, et al. 2013 classification criteria for systemic sclerosis: an American College of Rheumatology/European League Against Rheumatism collaborative initiative. Arthritis Rheum. 2013;65(11):2737-47.

19. LeRoy EC, Medsger Jr TA. Criteria for the classification of early systemic sclerosis. J Rheumatol. 2001:28(7):1573-6.

20. Clements $P$, Lachenbruch $P$, Siebold J, White B, Weiner S, Martin R, et al. Inter and intraobserver variability of total skin thickness score (modified Rodnan TSS) in systemic sclerosis. J Rheumatol. 1995;22(7):1281-5.

21. Ma Y, Mazumdar M, Memtsoudis SG. Beyond repeated-measures analysis of variance: advanced statistical methods for the analysis of longitudinal data in anesthesia research. Reg Anesth Pain Med. 2012;37(1):99-105.

22. Cuomo G, Abignano G, Ruocco L, Vettori S, Valentini G. Hypocomplementemia in systemic sclerosis [in Italian]. Reumatismo. 2008;60(4):268-73.

23. Hudson M, Walker JG, Fritzler M, Taillefer S, Baron M. Hypocomplementemia in systemic sclerosis - clinical and serological correlations. J Rheumatol. 2007:34(11):2218-23.

24. Senaldi G, Lupoli S, Vergani D, Black CM. Activation of the complement system in systemic sclerosis: relationship to clinical severity. Arthritis Rheum. 1989;32(10):1262-7.

25. Batal I, Domsic RT, Shafer A, Medsger Jr TA, Kiss LP, Randhawa P, et al. Renal biopsy findings predicting outcome in scleroderma renal crisis. Hum Pathol. 2009:40(3):332-40

26. Valentini G, Bencivelli W, Bombardieri S, D'Angelo S, Della Rossa A, Silman AJ, et al. European Scleroderma Study Group to define disease activity criteria for systemic sclerosis. III. Assessment of the construct validity of the preliminary activity criteria. Ann Rheum Dis. 2003;62(9):901-3.

27. Valentini G, D'Angelo S, Della Rossa A, Bencivelli W, Bombardieri S. European Scleroderma Study Group to define disease activity criteria for systemic sclerosis. IV. Assessment of skin thickening by modified Rodnan skin score. Ann Rheum Dis. 2003;62(9):904-5.

28. Yuan J, Chen M, Zhao MH. Complement in antineutrophil cytoplasmic antibody-associated vasculitis. Clin Exp Nephrol. 2013;17(5):642-5.

29. Muangchan C, Harding S, Khimdas S, Bonner A, Group CSR, Baron M, Pope J. Association of $\mathrm{C}$-reactive protein with high disease activity in systemic sclerosis: results from the Canadian Scleroderma Research Group. Arthritis Care Res (Hoboken). 2012:64(9):1405-14.

\section{Submit your next manuscript to BioMed Central and we will help you at every step:}

- We accept pre-submission inquiries

- Our selector tool helps you to find the most relevant journal

- We provide round the clock customer support

- Convenient online submission

- Thorough peer review

- Inclusion in PubMed and all major indexing services

- Maximum visibility for your research

Submit your manuscript at www.biomedcentral.com/submit
Biomed Central 